\title{
Classification, confusion and misclassification
}

The classification of objects and phenomena in science and nature has fascinated academics since Carl Linnaeus, the Swedish botanist and zoologist, created his binomial description of living things in the 1700s and probably long before in accounts of others in textbooks long since gone. It must have concerned human beings and animals since they first became aware.

Classification is designed to assist understanding. The response to a phenomenon, object or substance may be assisted by knowing which class the subject belongs to: a chemist may know what to expect from a reaction by knowing that an aldehyde, not an ester, is being investigated.

Classifications change and in that process, we can see that someone or some group has recognised that a previous classification hindered understanding or moulded the response to a finding incorrectly. This change is a beneficial event, but it tells us that at any given time we should question classifications to look for flaws.

In 2014, the World Health Organization's ${ }^{[1]}$ classification of endometrial hyperplasia changed and was simplified to include two categories, compared with four previously: from with atypia and without (each of those complex or simple) to hyperplasia with atypia or without atypia. The previous classification, which was suited to a pathologist's ability to distinguish, had led to confusion and mismanagement by gynaecologists. What is worrying if not the word 'complex'? But this is just a histological term. The important distinction was atypia.

The latest Royal College of Obstetricians and Gynaecologists (UK) guidelines on placenta previa and accreta, published in $2011,{ }^{[2]}$ recognise a change in the classification of placenta previa. Placenta previa was once classified into four categories: entering the lower segment, approximating the os, covering the os, or mainly in the lower segment. This has now been replaced by major and minor: approaching the os in the lower segment, or covering the os.

Why change? Because the elongation of the lower segment in pregnancy renders many previas insignificant, exposing overtreatment and needless anxiety when mere confirmation of 'emigration' was all that was necessary. Emigration is rendered less likely if the placenta covers the os by $1.5-2 \mathrm{~cm}$ or if it is anterior when there is a previous caesarean section.

But all is not yet simple: what remains confusing is the doubt, highlighted in the text, that surrounds the correct safe management in labour of a placenta that is classified as approaching the os. Many of the recommendations in the guideline relating to this problem are level 'D'. In out-patient managed antenatal cases in highly resourced settings, with good transport, the likelihood of heavy bleeding seems independent of the degree of previa. ${ }^{[2]}$ In other words, there is no clear evidence to support one course of action against another based on the identification of a particular case within this classification. The classification is incomplete, partly due to the difficulty of studying a sporadic problem scientifically or perhaps because of the difficulty, once again, of recognising subsets within the classification.

The guideline also details an important change in another classification: placenta accreta, the abnormal attachment of the placenta to the myometrium that may be associated with placenta previa. The risk of accreta is up to $40 \%$ in cases of previa with three previous caesareans. Incorrectly managed placenta previa may be cataclysmic, and even more so with accreta. But how to classify placenta accreta?
Previously it was said that accreta involved early myometrial invasion, increta further and percreta beyond.

Abnormal vasculature adjacent to the uterus on imaging may represent penetration of the placenta through the uterus or mere recruitment and enlargement of separate vessels beyond to enhance blood supply to the myometrium. The guideline discusses magnetic resonance imaging findings and Doppler findings (grey scale, colour and three-dimensional power Doppler) that cast light on the extent of invasion. The truth is, you cannot easily tell the extent of penetration.

If there is little invasion of the placenta you can tell, but if there is more, you cannot definitively say until the operation. So, if in doubt, take a senior surgeon, a cell saver (if available), and be prepared. Hence the simplification - it is all 'accreta'. The old classification is accurate to the pathologist, not the obstetrician.

Some classifications naturally evolve with time. Premature labour was once simply classified as being before 37 weeks. Modern care of the newborn makes the difference between delivery at 38 weeks, 37 weeks, and even 36 weeks most often meaningless. Hence the further classification into very preterm and extremely preterm newborn.

Similarly, the arbitrary cut-off of $2.5 \mathrm{~kg}$ as the classification of a low birthweight baby, malnourished in the uterus and at risk of longterm injury, has been replaced by the description growth restricted - a baby of $2.8 \mathrm{~kg}$ may be at risk if it was intended/capable of growing to $3.6 \mathrm{~kg}$, and such a fetus may not suffer the rigours of birth and delivery or even persisting pregnancy well. This failure to reach potential and be classified as growth restricted might be recognised clinically by poor symphysis fundal height development and palpated low liquor levels, which are unreliable, or asymmetric growth on sonar, which may not be available in all settings. The at-risk fetus, despite attempts to classify and identify, may still often go unrecognised.

The classification of vulval disease, too, has changed four times in 40 years. Clearly, something is not understood correctly.

The common woodlouse that inhabits the insect world is, in fact, a crustacean, which includes crabs, lobsters and prawns. It is a close relative and survivor of the ammonites, many now extinct, which once inhabited and occasionally still do inhabit the ocean floor. The woodlouse, however, has many segments and many legs, as do the myriapods, millipedes and centipedes that, like the woodlouse, inhabit the insect world.

Evidently, classification can help or hinder; it can make sense in one way but not in another and it can be confusing and misleading. Anyone who goes to the seaside to look for woodlice would be cruelly disappointed. There must be other similar confusions and misclassifications that inhabit the world of obstetrics and gynaecology.

\section{Will Edridge}

Editor

1. Emons G, Beckmann MW, Schmidt D, Mallmann P, for the Uterus Commission of the Gynecological Oncology Working Group (AGO). New WHO Classification of Endometrial Hyperplasias. Geburtshilfe Frauenheilkd 2015;75(2):135-136. https://doi.org/10.1055/s-0034-1396256

2. Royal College of Obstetricians \& Gynaecologists (RCOG). Placenta Praevia, Placenta Praevia Accreta and Vasa Praevia: Diagnosis and Management (Green-top Guideline No. 27). London: RCOG, 2011 https://www.rcog.org.uk/en/guidelines-research-services/guidelines/gtg27/ (accessed 29 April 2017). 